\title{
Renal function of neonates during gentamicin treatment
}

\author{
I TESSIN, J BERGMARK, K HIESCHE, R JAGENBURG, AND B TROLLFORS
}

Department of Paediatrics, Hospital of Mölndal; Pharmacia Diagnostics AB, Uppsala; Department of Clinical Chemistry and Department of Infectious Diseases, University of Göteborg, Göteborg, Sweden

SUMMARY Thirteen newborn infants, 8 term and 5 preterm (gestational age 31 to 36 weeks), were treated for between 3 and 7 days with gentamicin and ampicillin or cloxacillin because of suspected bacterial infection. The dosage of gentamicin was carefully monitored by serum concentration assays. Urinary alanine aminopeptidase, urinary $\beta_{2}$-microglobulin, serum urea, and serum $\beta_{2^{-}}$ microglobulin were measured during and after the end of treatment to detect signs of renal toxicity. Levels of urinary aminopeptidase increased in 12 of them, indicating damage to the cells of the proximal tubuli. Changes in urinary $\beta_{2}$-microglobulin followed the normal physiological course seen in neonates after birth. Serum levels of urea and $\beta_{2}$-microglobulin did not indicate any drugassociated depression of glomerular filtration rate.

In recent years it has been demonstrated that in most adults the kidneys are affected by therapeutic doses of gentamicin. After only one injection there is an increase in the urine of enzymes from renal tubular cells-for example, alanine aminopeptidase (AAP). ${ }^{1}$ In many patients there is also an increase in the urinary excretion of $\beta_{2}$-microglobulin, indicating a reduced reabsorptive capacity of the proximal tubuli. ${ }^{2}$ Moreover, a small but significant decrease of glomerular filtration rate (GFR), as measured by ${ }^{51}$ chrome-EDTA clearance, has been demonstrated. ${ }^{2}$ This decrease is accompanied by a significant increase in serum $\beta_{2}$-microglobulin, but not in serum creatinine.

Aminoglycoside-associated nephrotoxicity is considered to be rare in infants, but it is difficult to assess renal function and to determine which changes are drug associated and which are dependent on other factors. ${ }^{3-5}$ Clearance determinations may be difficult to perform in neonates. Serum urea, commonly used to approximate GFR, is influenced by the increased protein intake after birth. ${ }^{6}$ Furthermore, renal function in neonates alters according to gestational $\mathbf{a g e}^{6}$ and pronounced changes in both glomerular and tubular function occur after birth. ${ }^{6}$

The aim of this study was to search for signs of renal toxicity in neonates treated with gentamicin. Levels of urinary AAP and urinary $\beta_{2}$-microglobulin were used to evaluate effects on the proximal tubuli.
Levels of serum urea and serum $\beta_{2}$-microglobulin were measured to detect major changes in GFR.

\section{Patients}

Thirteen neonates, 6 boys and 7 girls, were treated with gentamicin. Eight were born at a gestational age of 37 to 42 weeks (term). Four were born at a gestational age of 34 to 36 weeks, and one was born at 31 weeks. The gentamicin treatment started within 24 hours of birth in 6 patients, between the 2nd and 5th day of life in 6 patients, and on the 13th day of life in one. Indications for treatment were febrile mother at birth (2 patients), purulent amniotic fluid (1), cutaneous abscess (1), and suspicion of septicaemia (9). Septicaemia was suspected because of cerebral irritation and cyanosis (4 patients), hyperthermia (3), hypothermia (1), and impaired respiration (1).

Gentamicin was given intramuscularly in a dose of $2.5 \mathrm{mg} / \mathrm{kg}$ every $18 \mathrm{th}$ hour. The dose was adjusted according to the serum concentrations of gentamicin assayed daily or every other day. Blood was taken immediately before (peak level) and one hour after (trough level) the injection. Peak levels below $4 \mathrm{mg} / 1$ were considered subtherapeutic requiring an increase in the dose.

The patient with cutaneous abscesses was given cloxacillin and the other patients received ampicillin 758 
in combination with gentamicin. In one patient Staphylococcus aureus was isolated from the blood. His condition soon improved on treatment with gentamicin and ampicillin, but from the 4th day only cloxacillin was given. One patient had a serious bacterial infection but blood cultures were negative. From the urine a Klebsiella $\mathrm{sp}$. was isolated (bladder puncture) and he had a thrombocytopenia (thrombocytes $26 \times 10^{9} / 1$ ) when treatment started. His condition soon improved with treatment which was given for 6 days. In the other 11 patients, all of whom improved rapidly, the suspicion of septicaemia was not verified by cultures and gentamicin treatment was stopped after 3 to 7 days.

\section{Methods}

AAP in the urine was analysed with a method using L-alanine- $\rho$-nitroanilid as substrate at $37^{\circ} \mathrm{C} .{ }^{7}$ Since no reference values of urinary AAP in neonates are available, AAP was analysed in urine samples from 20 healthy term neonates not receiving any drug. The mean urinary AAP activity was 0.097, 0.098, and $0.101 \mathrm{mkat} / \mathrm{mol}$ creatinine on the $1 \mathrm{st}$, 3rd, and 5 th days of life, respectively. The reference interval between the 2.5 and 97.5 centiles was $0.03-0.22$ $\mathrm{mkat} / \mathrm{mol}$ creatinine, as calculated graphically by a method described earlier. ${ }^{8}$

Beta ${ }_{2}$-microglobulin in serum and urine was measured by a radioimmunosorbent assay (Phadebas $\beta_{2}$-microtest, Pharmacia Diagnostics AB, Uppsala, Sweden). To avoid degradation of $\beta_{2}$-microglobulin in acid urine samples, $0.6 \mathrm{~mol} / 1 \mathrm{NaHCO}_{3}$ was added until the $\mathrm{pH}$ reached 6 . The urinary levels of $\beta_{2}$-microglobulin must be related to gestational age and to changes occurring after birth. The levels are lowest at a gestational age of 35 weeks and increase gradually to the 40th week because of increasing GFR with unchanged tubular reabsorptive capacity. ${ }^{9}$ During the first days after birth urinary $\beta_{2}-$ microglobulin increases further for the same reason. ${ }^{910}$ Finally, when the proximal tubuli have matured, urinary $\beta_{2}$-microglobulin decreases. ${ }^{9}$

Since urine was collected as random samples, urinary AAP and urinary $\beta_{2}$-microglobulin were related to urinary creatinine to eliminate differences in urinary flow.

\section{Results}

The trough serum levels of gentamicin were below $1 \mathrm{mg} / \mathrm{l}$ in most patients during the entire treatment time. Only on a few occasions were trough levels of between 1 and $2 \mathrm{mg} / 1$ observed. The peak levels of gentamicin were below $4 \mathrm{mg} / \mathrm{l}$ on one occasion in each of 3 patients. Apart from this the peak serum levels ranged from 4.3 to $8 \mathrm{mg} / \mathrm{l}$ and were generally between 5 and $6 \mathrm{mg} / 1$.
In the 8 term infants treated with gentamicin, the median activity of urinary AAP was $\mathbf{0 . 0 7}$ (range $0.06-0.14) \mathrm{mkat} / \mathrm{mol}$ creatinine before the start of treatment. In all of them the AAP activity increased during treatment. With only one exception did the AAP activity during treatment exceed the upper reference limit of $0.22 \mathrm{mkat} / \mathrm{mol}$ creatinine (Figure, left). After treatment ended the AAP activity decreased but did not reach the pretreatment level during the observation period. In the 4 infants born at a gestational age of 34 to 36 weeks the AAP activity increased during the gentamicin treatment (Figure, right). In one of these patients, in whom gentamicin serum levels were no higher than in the others, a pronounced increase in AAP activity was present after only 2 days of treatment, followed by a decrease the next day despite continued treatment. In the patient born at a gestational age of 31 weeks, the AAP activity was high before treatment started but fell during it (Figure, right).

The urinary levels of $\beta_{2}$-microglobulin varied considerably (range $0 \cdot 13-14.8 \mathrm{~g} / \mathrm{mol}$ creatinine) before the start of treatment.

Apart from 2 term infants who had low levels within 2 days of birth, the variations could be explained by differences in gestational age and age at start of treatment. The changes that occurred in urinary $\beta_{2}$-microglobulin during the gentamicin treatment (Figure) followed the normal physiological course after birth both for term and preterm infants.

The median pretreatment level of serum urea was $4 \cdot 3$ (range $2 \cdot 1-8 \cdot 2$ ) $\mathrm{mmol} / 1$ and of serum $\beta_{2^{-}}$ microglobulin 4.1 (range 2.7-6.4) mg/l. Serum urea, but not serum $\beta_{2}$-microglobulin, showed a

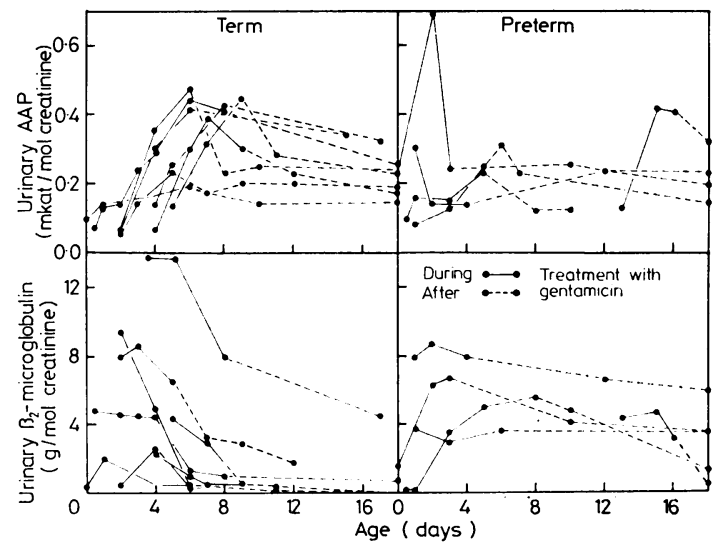

Figure Urinary alanine aminopeptidase $(A A P)$ and urinary $\beta_{2}$-microglobulin in 8 term and 5 preterm neonates during (full line) and after (broken line) treatment with gentamicin. 
transient rise in 2 patients in whom treatment was started soon after birth. In the other patients, changes in both serum urea and serum $\beta_{2^{-}}$ microglobulin were insignificant during the gentamicin treatment.

\section{Discussion}

Aminoglycosides accumulate in the cells of the renal proximal tubuli, where also the main histopathological lesions, associated with aminoglycoside nephrotoxicity, are seen. ${ }^{11}$ In this study determinations of urinary AAP and urinary $\beta_{2}$-microglobulin were used to detect a possible toxic effect on the proximal tubuli of newborn infants treated with gentamicin.

AAP is an enzyme, considered to be specific for the cells of the proximal tubuli. ${ }^{1}$ Our results show that an increase in AAP in the urine is almost unavoidable during treatment with gentamicin in neonates, as previously shown in adults. ${ }^{1}$ Increases took place during the first treatment days in 12 out of 13 gentamicin-treated neonates. This increase cannot be explained by the presence of underlying infection since most of the patients soon recovered and an analysis in retrospect indicated that most of them had not had septicaemia.

Despite the drug-associated increase in urinary AAP, indicating damage to the proximal tubular cells, no impairment of the tubular reabsorption of $\beta_{2}$-microglobulin could be found. However, a drug-induced effect on the urinary levels of $\beta_{2^{-}}$ microglobulin in neonates may be difficult to detect, since the levels vary widely in healthy neonates, ${ }^{10}$ and since appreciable changes occur after birth. In most of the term neonates studied here, urinary $\beta_{2}$-microglobulin decreased during the gentamicin treatment. This can be explained by increasing tubular reabsorption of the protein due to the maturation of the proximal tubuli after birth. ${ }^{9}$ The increases in urinary $\beta_{2}$-microglobulin present during gentamicin treatment in the preterm infants can also be explained by the normal changes in renal function seen after birth, ${ }^{9}$ and should not be regarded as signs of drug-associated nephrotoxicity.

In adults a depression of GFR during gentamicin treatment has been demonstrated by using ${ }^{51}$ chrome-
EDTA clearance. ${ }^{2}$ It was not possible to measure GFR by clearance determinations in this study. However, the stable levels of serum urea and serum $\beta_{2}$-microglobulin indicate that no major changes of GFR during the gentamicin treatment had occurred.

It is concluded from these results that gentamicin treatment in the neonatal period causes an increased release into the urine of AAP from the proximal tubular cells. The clinical importance of this finding must not be overestimated, since no other signs of nephrotoxicity could be detected.

\section{References}

1 Mondorf A W, Breier J, Mendus J, et al. The effect of aminoglycosides on proximal tubular membranes of the human kidney. Eur J Clin Pharmacol 1978; 13: 133-42.

2 Trollfors B, Alestig K, Krantz I, Norrby R. Quantitative nephrotoxicity of gentamicin in nontoxic doses. $J$ Infect Dis 1980; 141: 306-9.

3 Marks S, Marks M I, Dupont C, Hammerberg S. Evaluation of three antibiotic programs in newborn infants. Can Med Assoc J 1978; 118: 659-62.

4 Milner R D G. Gentamicin in the newborn. Postgrad Med $J$ 1974; 50: Supplement 7, 40-4.

5 McCracken G H, Jr, Chrane D F, Thomas M L. Pharmacologic evaluation of gentamicin in newborn infants. J Infect Dis 1971; 124: Supplement, 215-23.

6 Smith C A, Nelson N M. The physiology of the newborn infant, fourth edition. Springfield, Ill: Thomas, 1976: 485-91.

7 Unger T, Struck H. Zur Aktivitätsbestimmung von Peptidasen und Aminosäurearylamidasen. J Clin Chem Clin Biochem 1976; 14: 449-52.

8 Landahl S, Jagenburg R, Svanborg A. Blood components in a 70-year-old population. Clin Chim Acta 1981; 112: $301-4$.

- Aperia A, Broberger O. Beta-2-microglobulin. An indicator of renal tubular maturation and dysfunction in the newborn. Acta Paediatr Scand 1979; 68: 669-76.

10 Karlsson F A, Hardell L J, Hellsing K. A prospective study of urinary proteins in early infancy. Acta Paediatr Scand 1979; 68: 663-7.

11 Appel G B, Neu H C. The nephrotoxicity of antimicrobial agents (first of three parts). $N$ Engl $J$ Med 1977; 296: 663-70.

Correspondence to Dr Ingemar Tessin, Department of Paediatrics, Hospital of Mölndal, S-431 80 Mölndal, Sweden.

Received 5 April 1982 